\title{
Risk Assessment of Computer Numerical Control (CNC) Machine Service Quality
}

\author{
Moriarty Kevin \\ Mechanical and Industrial Engineering, New Jersey Institute of Technology, Newark, the United Sates \\ Email address: \\ kmm65@njit.edu \\ To cite this article: \\ Moriarty Kevin. Risk Assessment of Computer Numerical Control (CNC) Machine Service Quality. American Journal of Mechanical and \\ Industrial Engineering. Vol. 6, No. 1, 2021, pp. 7-16. doi: 10.11648/j.ajmie.20210601.12
}

Received: February 25, 2021; Accepted: March 9, 2021; Published: March 22, 2020

\begin{abstract}
The background of this paper stems from the uncertainties related to machine down time can have a very high cost to modern manufacturing organizations in the form of, productivity losses, product lead times, and poor-quality low product yields. The objective of this research is to review applicable risk management systems and identify the most suitable system for CNC maintenance service. Recurrent CNC machine malfunctions can result in major economic losses, poor customer service, and loss of business reputation. Many examples of the occurrences of down time disruption due to machine failure and or maintenance issues are prolific throughout modern organizations. These instances cause process volatility, disruptions to productivity, digital incidents throughout the Industrial Internet of things (known as IIoT) process, which are all contributors to CNC machine down time risk. While "just-in-time" and lean concepts may benefit the organization in general, these concepts may contribute to risk during process stoppages. The identification, assessment and management of risk is the focus of this article. A combination of modern methods and techniques are presented for comparison to identify the most relevant method to use in this research. The method identified in this case is the Analytical Hierarchical Process (AHP) which is then used as the as the method of choice for this research to successfully address and manage the consequences of risks associated with CNC machine down time. The results of this research utilize the selected risk management system of AHP analysis and priority matrix methods. This is then simulated in the model of potential occurrences of variable risks. The estimation of prospective risk factor effects is identified and analyzed for the CNC production process. The outcomes of this research have identified the most appropriate method and tailored it to the $\mathrm{CNC}$ maintenance service, therefore enabling the user to execute the quantifiable risk assessment and management techniques.
\end{abstract}

Keywords: CNC, Quality Service, Risk, AHP, Life Cycle, Prediction

\section{Introduction}

Due to potential disruptions to process flow, many scholars have developed models and methods for managing risk. This research is primarily demonstrating a unique application of Analytical Hierarchy Process (AHP) Risk Assessment method to $\mathrm{CNC}$ service maintenance utilizing approaches used in other industries, but never applied to the CNC service industry. The area of supply chain risk and management has been responsible for a great deal of work subject material. These methods are reviewed and compared to the application of AHP in the CNC service maintenance field. Supply chain risk management known as (SCRM) utilizes a phased systematic method of identifying, assessing, prioritizing, monitoring, and mitigating potential disruptions to the supply chain and this method can also be applied and utilized in the $\mathrm{CNC}$ machine tool space in order to reduce the negative impact and disruptions in production operations [3]. The likely risks and factors in manufacturing can be determined in a risk identification phase followed by the prioritization of risks through estimating the likelihood of occurrence and impact on the manufacturing process in the risk assessment phase. In an attempt to mitigate risk, the most appropriate mitigation strategy for risk or combinations of risks can be identified in a mitigation phase [20].

Over time, there have been a wide variety of debates about whether risk is a subjective or objective occurrence, both have their negative and positive consequences. The 
relationship between risk and uncertainty have raised a wide range of debates. The subjective view supports a belief that risk is determined by the views of the interested party and participants involved (political, industrial community or the general public as well as other views). There are others who consider risk objectively defined based upon quantitative data and analysis. While most agree with the outcomes, risks are usually associated with undesirable effects (i.e., losses). While incidents may be relatively uncommon, researchers focus less on the potential positive effects (i.e., benefits) in uncertain situations. Regarding the relationship between risk and uncertainty, one may consider risk as the expected outcome of an uncertain event, while a different perspective may view risk as the uncertainty of events and possible outcomes. This model views the knowledge of decision makers for the entire supply chain, from complete security to fundamental uncertainty, [27].

There are different stages of increasing uncertainty between the two. The closest thing to complete certainty is a situation that is the basis of a typical SCRM: in this case, the supply chain structure is fully known, and the possibility and impact of future events are known based on objective probabilities, [4]. This is the opposite of the next more uncertain level, where these probabilities are unknown and SCRM can only rely on subjective beliefs. While the supply chain risk management process has been extensively researched, that has not been the case for $\mathrm{CNC}$ machine service and research into risks associated with machine down time. A process of Risk Assessment for $\mathrm{CNC}$ machine service certainly benefits from an objective rather than a subjective process, as long as the focus is based upon quantitative data items in a deterministic model. This introduces a new body of knowledge to the service operation and process.

From the view of risk management for the purpose of controlling or completely avoiding the negative effects of risk means negative effects will be minimized and/or eliminated. The result would be improving productivity and profitability of the $\mathrm{CNC}$ machining operation. This research has been addressed in the areas of Supply Chain Risk Management as well, "After all, SCRM is only effective if the structure of the supply chain is at least fully understood". [4].

\section{Classification Models}

\subsection{Problem Evaluation}

Computer Numerical Control (CNC) machining is an integrated process with several different manufacturing business categories. These manufactures are quite diverse and spread over numerous products and markets, which could be consumer products, aerospace components, medical equipment parts, etc. These manufacturers work to coordinate, convert, and process raw materials into a final product [5].

The customer participates in the $\mathrm{CNC}$ downtime risk and an alternative from a customer perspective is that machine down time will cause delays in parts made by the $\mathrm{CNC}$ machines, whereas the objective is to fulfil a customer request. [12]. Since the late 1990s there has been an increasing focus on the performance, design, and analysis of $\mathrm{CNC}$ machine down time, and the manufacture of machined parts is an integral part of this supply chain [5].

The purpose of a CNC machine down time analysis is to maximize profit in a process of generating value for the customer [12]. In other words, the analysis purpose is to maximize the difference between the final product worth and the cost incurred by the CNC machine down time in order to deliver the product to the customer on time and within cost constraints. The information gained by the analysis can be used in the work process of managing $\mathrm{CNC}$ machine down time, where the aim is to increase competitiveness [23]. Furthermore, this research maintains that continuous work on management of CNC machine down time will help visualize how the $\mathrm{CNC}$ machine productivity and affects part production and distribution but is not always an isolated aspect of the supply chain. This research also maintains, that in order to deal with productivity problems, having knowledge of costs and stock levels are not adequate enough to address downtime productivity issues, since no quantitative model can capture all aspects.

Economic systems are experiencing increasing uncertainty and complexity [16]. A shift in risk management has occurred and companies now render more informed decisions with the aid of risk: assessment, control and mitigation tools. This position is also presented by authors [13], who contend that weaknesses within the supply chain are the results of increases throughout competitive global environment. Svensson contends that dependencies between organizations will create vulnerable situations and companies will need to address these with the assistance of risk management and risk assessment [24]. When addressing risk assessment there are two views to consider according to Gaudenzi, \& Borghesi, which are:

1. Risks occur at various intervals, inside the company as well as at a network level [13].

2. The estimation of risk is vastly subjective, due to analyst's individual concept of a significant risk [13].

The use of lean techniques and timely Just-in-time concepts in production and logistics can improve efficiency. However, since the supply chain leaves little room for errors and changes, the supply chain is susceptible to adverse events [3]. Additionally, companies are becoming more global and their degree of vertical integration is decreasing, which is thereby increasing the complexity of the supply chain and exposing it to more risk [6]. This very issue now exists within the $\mathrm{CNC}$ machining manufacturing environment as well. Finally, countless incidents that disrupted the global supply chain and have been seen in recent times, which have attracted worldwide attention. For example, such things as natural disasters (the tsunami and floods in Japan 1 that caused a global shortage of many products from that region, to man-made disasters (like the 9/11 terrorist attack). This 
also includes the current 2020 global economic crisis caused by the COVID19 pandemic, leading to health, financial and economic instability, not to mention the political consequences

While Supply Chain Risk Management (SCRM) this is a well-known practice within the logistics community, which encompasses research of many different methods, from qualitative methods (such as empirical research and conceptual theory) to quantitative methods (such as linear programming optimization, data analysis, and mathematical modeling). Regarding implemented strategies, SCRM can employ passive or active strategies: the former is applied after risks have arisen, while the latter allows identification and assessment before risks arise in order to develop appropriate mitigation and contingency plans, [4]. The novel aspect of this research is that these very topics now add to the body of knowledge within the areas of CNC machining which this research has identified as not currently being employed and this research is developing the application of these concepts.

Recent events such as the KFC chicken supply crisis in early 2018 have further demonstrated the view that weighing risks and developing contingency plans before major changes in the supply chain can potentially prevent major losses [15]. To achieve this goal, proactive strategies rely on the ability to accurately predict the likelihood of occurrence and the impact of potential risks. A wide range of technologies can be used to achieve this necessary predictive capacity. [4].

Financial risk assessment is another common area of the application of risk assessment. Forecasting methods play an important role in the development of early warning systems for financial crises. The use of several different algorithms (e.g., NN, Decision Tree and SVM) to predict the financial distress of a group of companies based on 31 financial indicators [14]. Recently, predictive models for intentional and unintentional financial adjustments have also been developed [8]. They use a variety of classification algorithms (such as ANN, decision trees, naive Bayes, support vector machines, and Bayesian belief networks) [8]. Other methods also used in research have been decision trees, support vector machines (SVM), neural networks (NN), and logistic regression to collect data from 100 US companies that went bankrupt and compared the performance of these algorithms [26]. Likewise, used methods for classifying the neighbors with weighted voting relationships to predict the insolvency probability of SMEs based on financial and non-financial data.

Risk usually originates from a lack of information, and its identification is an intricate and costly process due to the high uncertainty of event occurrence and difficulty in collecting and analyzing the risk data [18].

\subsection{Problem Background}

The circumstances surrounding necessary service; corrective, preventative, and predictive, and how to structure the service maintenance process. The choices are to divide the function into three categories: $i-$ in house (SM-1) service, ii- service contract (SM-2), and iii- third party service providers (SM-3).

i. capabilities of the SM-1 are quick response on routine repair and maintenance, while maintaining minimal repair part inventory

ii. capabilities of the SM-2 are reasonable response time and additional capabilities for specialty and larger scale repairs and maintain a larger replacement parts inventory and part supply network (and/or warehouse).

iii. capabilities of the SM-3 are more final recourse when the service is beyond the scope of SM-1 \& SM-2. This is critical when it requires specialties which only a third part service supplier is capable of and the impact of the risks associated with the service event will potentially have a serious impact on the organization and productivity. This has a strong impact upon the inventory and warehousing maintenance function by requiring less storage of spare part quantities.

An important technical challenge is to identify the potential risks posed by new product launches and the strategies to mitigate those risks. Sources of these potential risk factors can be from within the organization internal; technical abilities, human resources, suppliers, and stake holders, or external; supply chain partners, such as third-party suppliers, customers and service providers as well as competitors, and political, social or environmental forces

This implies that an effective risk analysis should not only focus on the total lifecycle stages of a product but extends to multi-lifecycle material flow, [10]. Qualitative and quantitative capabilities for modeling and evaluating product design risks have been addressed in literature, [10]. However, the models developed in the literature have nothing to do with determining the functions available for optimal product configuration design, making them less reliable in decision making. Effective decisions for sustainable product design must incorporate risks and uncertainties into the optimization of the product configuration design, [11]

\section{Research methodology}

\subsection{Purpose}

The point of this research is to develop a quantitative method of assessing risk within the CNC machining operation by integrating multiple activities. Examples consist of: identifying risk indicators, collecting, and storing risk data, converting risk management problems into the Analytical Hierarchy Process (AHP), and identify information using the AHP to analyze and interpret the results. Risk mitigation strategies. To implement this framework, knowledge of interdisciplinary areas and several methods of data collection and evaluation are required. 
Literature on Supply Chain Risk Management, relating to logistics and information management system were reviewed to assess comparative models. Key steps in the overall AHP process are; identifying the risk management team and choosing the hierarchal components, which are critical roles in developing this complex model. The conceptual model was developed refined and verified using simulation data in excel. The type, severity and frequency of the risks vary depending on several factors associated with the CNC machining process. CNC Machining operations vary, and each has its own risk profile and risk components. Therefore, the tests propose varying frameworks in order to gain important insights, of the operation.

\subsection{CNC Body of Knowledge}

An addition to the $\mathrm{CNC}$ operational body of knowledge making this research novel and unique is to integrate this research into both the $\mathrm{CNC}$ machine controller as well as the with ERP (Enterprise Resource Planning) software in order to integrate service within the processes already incorporated within ERP (personnel, procurement, production, R\&D, sales, transport, finance, etc.). Through this process now CNC service/maintenance can be integrated into one platform and thus improve process management to gain competitive advantage. Hence, the AHP method was chosen to quantify service risk in this case.

A point that the evaluation of risk is subjective also indicates that there are also several different definitions of what $\mathrm{CNC}$ machine down time risk could be. Investigating comparisons of risk definitions has helped come up with a definition that should cover the entire manufacturing machining process [17]. The definition of risk from this review which can be applied to $\mathrm{CNC}$ machine down time is as follows, "the likelihood and impact of unexpected macro and/or micro level events (interruptions/failures) or conditions that adversely affect any part of a CNC machine (down time) leading to operational, tactical, or strategic level failures or inconsistencies within the product/process flow "[Ho et al., (2015, s. 4)]. It must also be clear that there is a difference between uncertainty and risk. Risk is the perceived possibility of events and can be represented by measured probabilities in a given situation. Uncertainty is when the possibility of events cannot be measured due to the impreciseness of a given set of circumstances [9].

\subsection{Risk of Down Time}

CNC machine down time risks and risk factors may be identified in several ways. This may all be in the eyes of the observer [13]. What this implies is that risk should be connected to the objective of CNC machine down time reduction to facilitate selection and grading of different risks.

Risk assessment is an untapped research resource of improved productivity and profitability that should be highly connected to the objectives of manufacturing machine operations. This is the very aspect of this research that makes it so unique in the CNC machining environment. The amount to which these objectives are accomplished is dependent upon the magnitude by which the uncertainties within manufacturing exists [16].

Some studies have tried to incorporate "soft values" to risk assessment into optimization problems in an attempt to further develop a realistic solution to a wide range of different problems which may apply to CNC machine down time risks.

\subsection{An Optimization Model}

for the time sensitive products has been created, which may also be applicable to $\mathrm{CNC}$ machine down time. The model aims to solve an allocation problem by minimizing risk and cost throughout the manufacturing process [22]. This model could be reconfigured to be applied to different problems. In case, a general network optimization model with integrated risk assessment would need to be developed for the risks associated with CNC machining.

In this study it is intended to assess multiple service risks which can interrupt product process flow from manufacturing to finished goods (customer), considering cost and risk. This will help develop the AHP model that helps manufacturing personnel choose the best process flow for machining operations based upon risk factors. This will be accomplished by investigating the following topics:

1. What risks exist within the different process flows?

2. What are cost variations through different process flows?

3. How is risk assessed and compared to costs as different process flows are evaluated?

\section{Model}

\subsection{Distinct Application for CNC Maintenance}

This model is a unique adaptation of risk assessment tools applied to CNC machine down time which has never been applied to the fabrication cycle before. It is not designed to help solve or minimize identified risks. The objective of the model is to reveal the risks and costs to the organization and assist with making well informed decisions so unnecessary risks and costs can be minimized.

The model results in an analysis utilizing spread- sheets simulation for the risk model which outlines the likelihood (in terms of number of possible events in a given time frame; for example, 1 in 5 years,) and duration (estimated using a triangular distribution due to its intuitive nature) of each disruption category A process flow diagram has been developed to display the phases of service maintenance. This diagram illustrates the levels of service provided while identifying the relationships between the different levels and the inputs and output controls and resources associated with the service process.

Figures $1 \& 2$ below show an overview of the hypothetical distribution channel setup. 
The model does not solve the risk related problems, merely the identification of the potential existence of the risks and the analysis of relative differences in risks between production process flows. The study does not include model integration or any practical data, but the development of simulation ad-hoc data is implemented. To increase validity, we use a pilot random generator of data parameters within the model. Consequently, the reliability of the model results is harder to assess for this method. The evaluation of risk perceived within model for $\mathrm{CNC}$ machine down time is highly subjective, which could lead to not taking a critical enough approach to areas of improvement and reliability within the production environment.

CNC Service Risk Flow Chart; reference example -

A risk management phase model. This process consists of Layers 0,1 and 2 ; with additional Layers possible; $n, m$, ..etc.

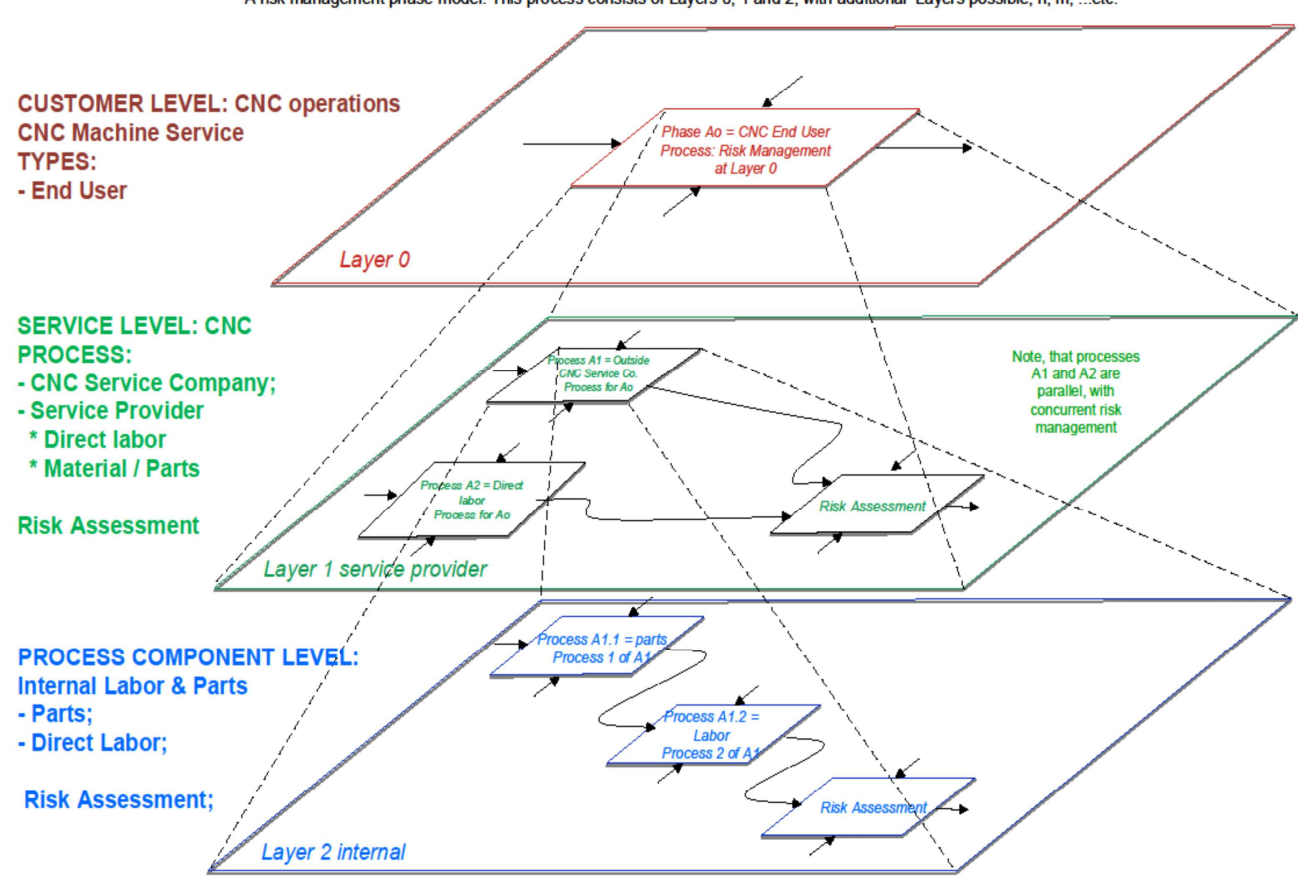

LEVELS: 2 - 0: Organizations implement risk assessment and management practices throughout these levels

Figure 1. System Risk flow chart.

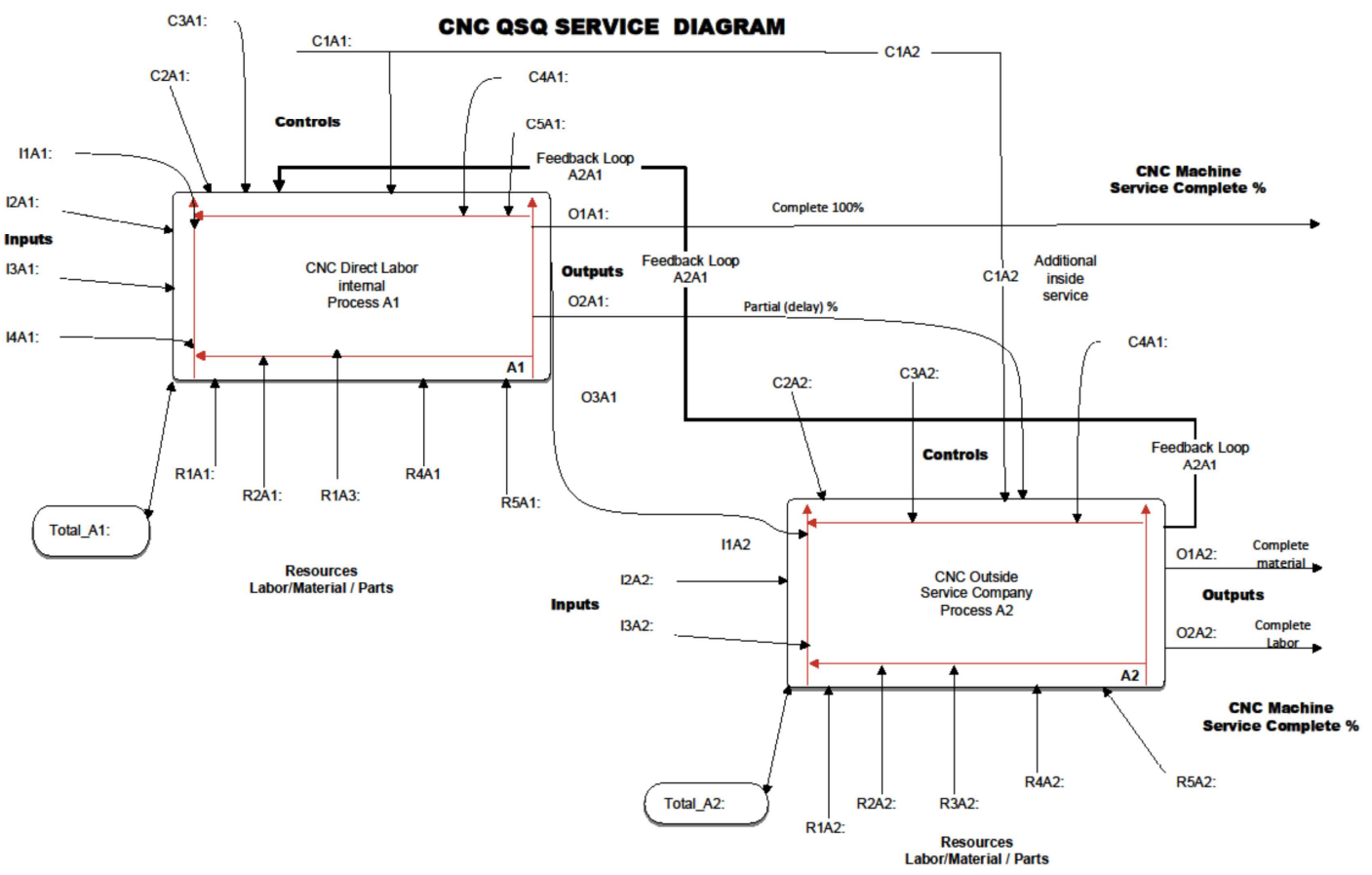

Level 1 \& Level 2 QSQ service system diagram Ref.: Sample Service, Date: Xxx 00, 2020, Version: Ver. 1.0

Figure 2. System service diagram. 


\subsection{Researched Alternative Models}

\subsubsection{Data Mining (DM)}

In order to develop a data-driven framework to $\mathrm{CNC}$ down time risk, the key principles of data mining (DM), data warehouse and risk management are systematically integrated. Figure 1 shows a step-by-step method for developing a DM-based framework. The core phases of the proposed model are: (i) identification of risk indicators, (ii) development of a risk data warehouse to collect and store risk data, and (iii) inclusion of a DM module that converts risk management problems into DM problems and explains risk management analysis results measured, [21].

\subsubsection{Multi-Criteria Decision Making (MCDM}

A popular concept that aims to find the most qualified alternative from a range of alternatives based on a range of criteria. These are techniques that can be used for various decision-making processes within management, business, society, and other fields. MCDM has two technologies.

There has been a realization that most models in the past were based on deterministic factors. In reality, however, it is difficult to know the exact production capacity and demand impact risk will have upon the operation. For these reasons, the uncertainty of the risk factors must also be taken into account.

While the risk factors of production and procurement problems are usually reviewed and managed as separate problems, there are many similarities in the fundamental issues. In addition, the uncertainty of the parameters of production and procurement are not addressed as thoroughly as other aspects of an organization as they tend to be more pragmatic and inconsistent, [19].

\subsubsection{Integer Non-Linear Program (MINLP).}

Another model developed for this purpose is Mixed This model takes into consideration all the production and procurement constraints to procure dependent items to meet independent items from the selected suppliers and their carrier to maximize revenue as well as to minimize procurement and costs. [19].

\subsubsection{Mixed Integer Linear Programming (MILP)}

This is an additional mathematical model for optimization is for optimization Industries [29]. Heuristic methods are also used to model problems in SSCM. [28]. The results show a data-driven model that predicts the optimal decisions with important accuracy, time efficiency, and flexibility to simultaneously handle several uncertainty sources disregarding their distributions, [7].

\subsubsection{Heuristic Use of Qualitative Methods}

Final methods to be considered are a heuristic in nature and use qualitative information to develop a quantified solution. The basic premise is that human technology is based on human preferences, such as: Analytical Hierarchy Process (AHP) and Best Worst Method (BWM), [1]. The BWM method has a significant limitation as this method restrictions the analysis to only two choices. This may skew the results depending on the gap between the two points of information. For this reason, this technique is not a preferred method for this research.

\subsubsection{AHP Method of Choice}

The chosen method in this research is AHP, because of the ease of development within a production environment and the use of operational knowledge to develop a solution based upon the wide variety of relevant information necessary to address the risk within $\mathrm{CNC}$ machine service.

Some studies of 4.0 industry have provided illustrations that offer economic examples which allow for the evaluation of productivity and technical efficiency of regional manufacturing industries, [7]. Results shown in research demonstrate that for both the standard model and the proposed factorial model, the economic theory is validated in terms of the importance of the inputs that form the manufacturing outputs, [7],

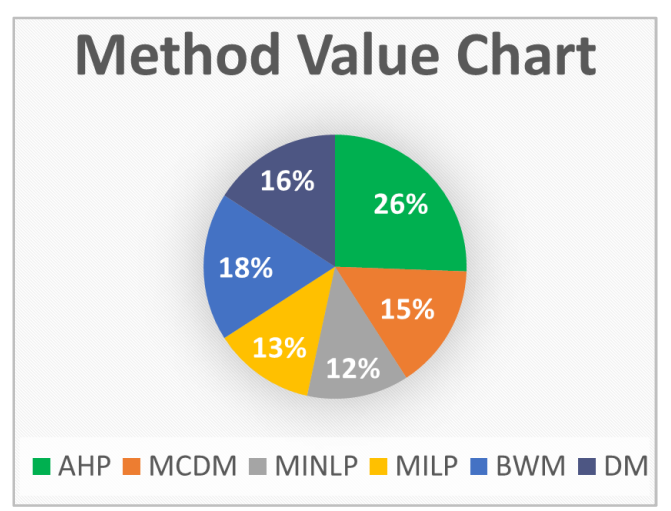

Figure 3. Method comparison value chart.

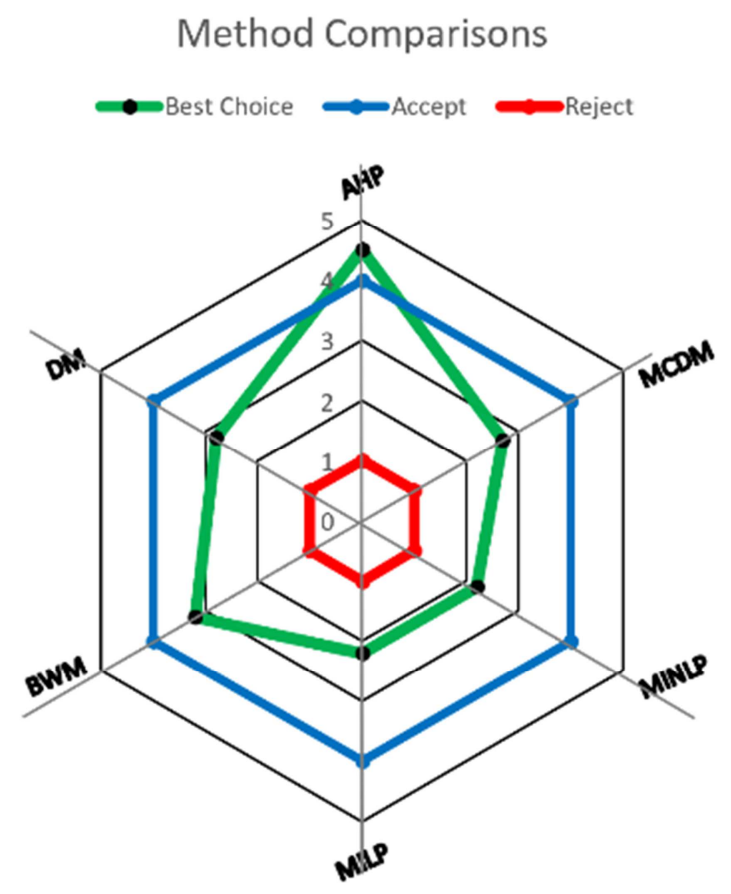

Figure 4. Method comparison Spider chart. 


\section{Quantitative Method Analysis}

In this section the method of analyzing quantitative information is presented in the article. In particular, the analytical hierarchy process (AHP) is discussed and developed.

Analytical Hierarchy Process (AHP) enables an organization to assemble and configure the identified risk components impacting the decisions process in a ranked format from top down in an analytical process of criteria, sub-criteria, and alternatives [25]. The creation of the hierarchy starts with the measurement step of the criteria and then moves down to the sub-criteria level. An application of AHP to the CNC machining processes is a novel approach to minimizing machine down time and fabrication interruptions.

A link should be established between risk and cost of the steps in the process. Cost may be integrated into the risk AHP to address this subject. Addressing this item of risk and cost relationship results in identifying risky criteria during $\mathrm{CNC}$ machine service. Once this is done the more specific risk sub-criteria aspects of this analysis are fully identified and integrated into the model to understand how these subcriteria can affect results of process. Costs are inherently connected to alternatives therefore cost is not broken down into further sub-criteria.

Different aspects of identified risk must be assessed as well to determine the potential impact on the service process flow when making the decision of what service alternative should be implemented. This is not necessary for the cost aspect as these are associated with the risk component, and not directly associated with the product cost, or the quantitative quality of the service.

After the construction of the AHP hierarchy, the element priorities need to be determined. This is done by establishing the parameter range of the different elements for a given criteria and assigning a value for the criteria for each element.

Heuristic values between $0<\mathrm{x}<5$ are chosen for the following comparisons.

1. Between criteria with respect to goal,

2. Between sub-criteria with respect to goal

3. Between alternatives with respect to sub-criteria

4. Between alternatives and elements

The comparison of the alternatives with regard to cost criteria is not done, as this is a process which can be done numerically when the costs of the different flows are calculated.

Every value between the $n$ criteria will create an " $x$ " and a corresponding inverted value $1 / x$ to create a $n * n$ matrix in example below figure 5 ,

$$
\left[\begin{array}{lllll}
\mathrm{x}_{11} & \mathrm{x}_{12} & \mathrm{x}_{13} & \mathrm{x}_{.} & \mathrm{x}_{1 \mathrm{n}} \\
\mathrm{x}_{21} & \mathrm{x}_{22} & \mathrm{x}_{23} & \mathrm{x}_{.} & \mathrm{x}_{2 \mathrm{n}} \\
\mathrm{x}_{. .} & \mathrm{x}_{. .} & \mathrm{x}_{. .} & \mathrm{x}_{.} & \mathrm{x}_{. .} \\
\mathrm{x}_{\mathrm{n} 1} & \mathrm{x}_{\mathrm{n} 2} & \mathrm{x}_{\mathrm{n} 3} & \mathrm{x}_{. .} & \mathrm{x}_{\mathrm{nn}}
\end{array}\right]
$$

Figure 5. Criteria matrix.
Next, we create the priority vectors. This is the Eigen vector of the matrix figure 6 . This can be manually calculated but is shown here using a computer solution (MS Excel). Using the example matrix in figure 6, each row represents one of the four criteria:

\begin{tabular}{l|c|c|c|c|} 
& \multicolumn{1}{c}{$\mathrm{A}$} & \multicolumn{1}{c}{$\mathrm{B}$} & \multicolumn{1}{c}{$\mathrm{C}$} & $\mathrm{D}$ \\
\cline { 2 - 5 } Criterior 1 & 1.00 & 3.00 & 5.00 & 2.00 \\
\cline { 2 - 5 } Criterior 2 & 2.00 & 1.00 & 1.00 & 3.00 \\
\hline Criterior 3 & 3.00 & 1.00 & 4.00 & 2.00 \\
\cline { 2 - 5 } Criterior 4 & 1.00 & 2.00 & 4.00 & 1.00 \\
\cline { 2 - 5 } & $\mathbf{7 . 0}$ & $\mathbf{7 . 0}$ & $\mathbf{1 4 . 0}$ & $\mathbf{8 . 0}$
\end{tabular}

Figure 6. Criteria values.

The first step is to normalize the matrix; this is accomplished by dividing each column component with the columns sum. This will make the new sum of each column equal to 1 . This results in the Matrix in figures $7 \& 8$ :

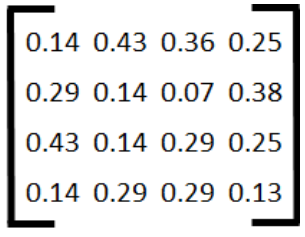

Figure 7. Eigen vectors.

\begin{tabular}{l|c|c|c|c|r} 
& \multicolumn{3}{c}{ Normalized } & \multicolumn{1}{r}{ Priority } \\
\cline { 3 - 6 } & A & B & C & D & \\
Criterior 1 & 0.14 & 0.43 & 0.36 & 0.25 & $\mathbf{2 9} \%$ \\
Criterior 2 & 0.29 & 0.14 & 0.07 & 0.38 & $\mathbf{2 2 \%}$ \\
Criterior 3 & 0.43 & 0.14 & 0.29 & 0.25 & $\mathbf{2 8 \%}$ \\
\cline { 2 - 5 } Criterior 4 & 0.14 & 0.29 & 0.29 & 0.13 & $\mathbf{2 1 \%}$ \\
\cline { 2 - 5 } & 1.0 & 1.0 & 1.0 & 1.0 & $100 \%$
\end{tabular}

Figure 8. Excel version of Eigen Vectors.

To calculate the priority vector, we use the average of each row, since we normalized the matrix the sum of elements in the vector will be equal to 1 . This process of normalizing the matrix is accomplished through the formulation of this procedure within the excel spread sheet developed for this purpose.

We get the following priority vector:

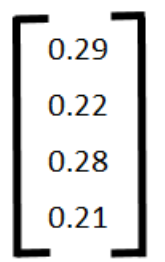

Figure 9. The Priority Vector.

In this example the criteria 1 has a relative weight of $29 \%$, the criteria 2 a relative weight of $22 \%$, criteria 3 a relative weight of $28 \%$ and the last criteria 4 a relative weight of $21 \%$. As demonstrated here this is a superior method of prioritizing risk values associated with the CNC service maintenance process because it quantifies the utilization of 
current traditional subjective and qualitative methods in use today in CNC service maintenance risks. This unique method is used to apply an acceptable mathematical quantitative analysis tool to the risk assessment and management of CNC service operations.

When analyzing the result, you should look at the consistency of the comparisons. In this study we evaluated the consistency index from the simulated values and compare it to the Random Consistency Index displayed in table.

The consistency index (CI) is calculated by the AHP criteria by dividing the minimum number of criteria (in this case 1$)$ by the actual total number of criteria $(4 \times 4)$ used in this analysis. Therefore, $\mathrm{CI}=0.0625$

Table 1. Random Consistency Index.

\begin{tabular}{lllllllllll}
\hline $\mathbf{n}$ & $\mathbf{1}$ & $\mathbf{2}$ & $\mathbf{3}$ & $\mathbf{4}$ & $\mathbf{5}$ & $\mathbf{6}$ & $\mathbf{7}$ & $\mathbf{8}$ & $\mathbf{9}$ & $\mathbf{1 0}$ \\
\hline $\mathrm{RI}$ & 0 & 0 & 0.58 & 0.9 & 1.12 & 1.24 & 1.32 & 1.41 & 1.45 & 1.49 \\
\hline
\end{tabular}

$\mathrm{n}=$ occurrence, $\mathrm{RI}=$ random index

Based on the number of criteria that we use; the random consistency index increases in value. When we have all of the elements, we use the consistency index (CI) given by the AHP excel program used and compare it to the random consistency index (RI) in order to calculate the consistency ratio (CR). This is done accordingly:

$$
\mathrm{CR}=\mathrm{CI} / \mathrm{RI}
$$

$\mathrm{CR}=0.0625 / 0.90=6.9 \%$, therefore this assessment is consistent. If $\mathrm{CR}$ is below $10 \%$ the subjective evaluation is considered consistent [25].

\section{Discussion}

In general, it is widely agreed upon that during the process of CNC maintenance some form of risk analysis is not only valuable, but necessary. Quantitative Risk Management is the process of applying quantifiable Quantitative Risk Analysis (QRA) results determined during a risk assessment into useful information for the management of risk within the organization. This is commonly accomplished by assigning impact scores, rankings, and probabilities to the subjective and qualitative inputs from the most knowledgeable stake holders. Then a variety of mathematical processes are applied in order to acquire a value for risk severity [2]. This process of quantitative analysis can then be applied to both costs and schedules.

Typically, in project management, schedules are managed by systems such as Critical Path Method, (CPM) networks, Project Evaluation and Review Techniques (PERT) analysis, or Monte Carlo Simulation. Quantitative analysis of cost is usually a culmination of the individual tasks or "work breakdown structures" (WBS) of the project. Then an analysis of the estimated time and impact of delays can be conducted using simulation methods e.g., Monte Carlo analysis, or analogous analytical techniques. Some observed practical studies have been done in this area of risk assessment and response planning. One organization that has devoted a great deal of effort to the subject is "The Project Management Institute" (PMI). Risk planning based upon established risk response planning, encompasses the determination of best practices and most effective mitigation methods (Project Management Institute, 2000).

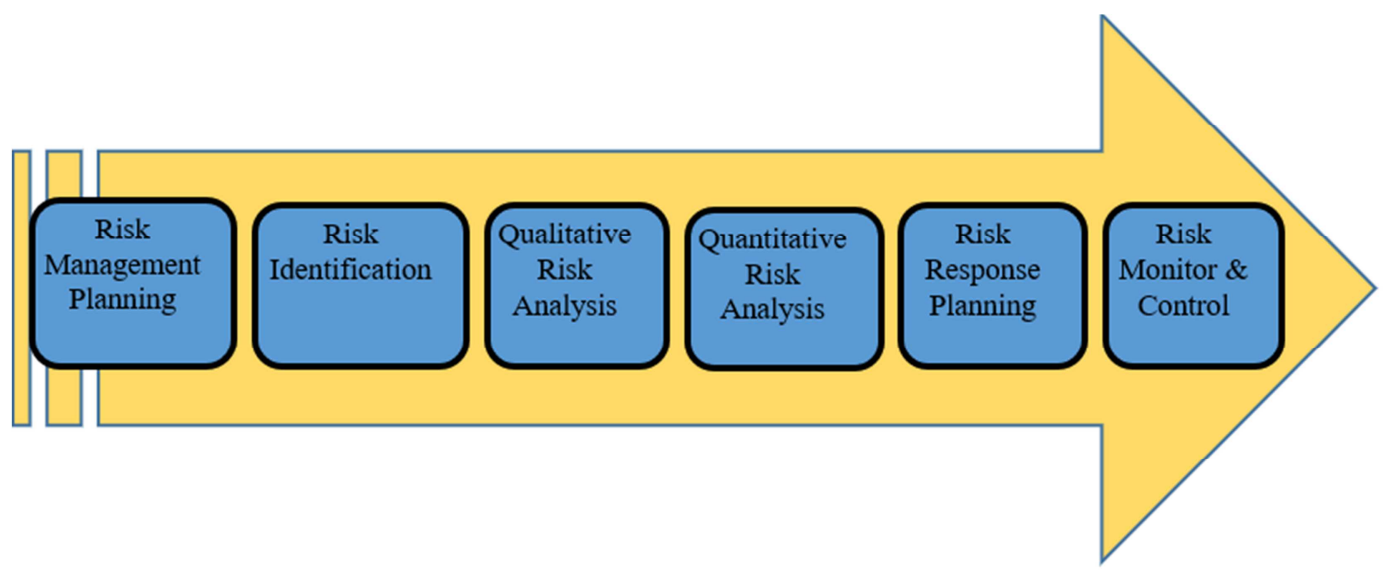

Figure 10. Risk Management Process Flow [PMBOK, (2014)].

The process of Response Planning can usually be performed after the qualitative stage or may also be accomplished after the quantitative stage. Either stage is acceptable due to the fact that maintenance goals will vary based on current circumstances and may be dependent upon the impact on the risk planning.

In the risk-response planning phase avoiding a risk is the key objective, for example availability of necessary replacement parts is key and that availability may fluctuate from time to time. Availability of key resources or capacity may also assist and can ensure the reduction of a risk.

These steps are performed in a hierarchical style as presented earlier in this paper under the Quantitative Methods section utilizing AHP. This process is developed in a group setting of key contributors. In the $\mathrm{CNC}$ production area, the risks having the greatest impact on down time and production interruptions should be addresses first. One of the most effective methods of analyzing risk is through the use of historical machine and maintenance data using machine monitor and control systems. 
The last step in this process evaluating and managing risk is risk monitoring and control. This requires, persistent checks, reexamination, scrutiny and surveillance to ensure no variable changes have occurred or have been introduced into the process. Changes within the organization internal or external may impact the circumstances of how the steps in the plan are affected. To accomplish this function an individual or team should be assigned the task of checking the process by inspection, survey or questionnaires of key people, and responsible roles within the organization, e.g., plant managers, department manages and CNC operators. This must be a continual process requiring comprehensive record keeping and regular reporting.

While it is true that modern maintenance programs have effectively improved the efficiency of operations, efforts to introduce changes to service quality have gone unchanged for a long time. Most service systems have not been as successful in improving the operational productivity because of the complexity of machine maintenance and a lack of full understanding of the unique aspects of highly engineered technical equipment i.e., $\mathrm{CNC}$ machines.

The integration of an automated service data system utilizing unique quantitative methods to identify, assess and prioritize machine maintenance risks has disclosed a significant opportunity to enhance the risk assessment and management of service-related interruptions within the organization.

This research identified, examined, and develop a modern systematic method of addressing risk assessment and management.

The AHP analysis and priority matrix methods are identified as key aspect of this process and an excel simulation approach has been used to model potential occurrences of variable risks when estimating the prospective effects identified risk factors may have on the production process.

The excel simulation successfully ascertained the priority values for the risk criteria identified in the model and the impact on the process. This has now given the CNC service environment a tool to assist in the improvement of product quality, productivity and reducing the impacts of production downtime and interruptions.

In summary, even though there has been a great deal of study conducted on the topic of risk throughout may industries and areas of interest to the best of my knowledge there has been no quantitative research into risk management of $\mathrm{CNC}$ maintenance service quality and the prioritization of criteria in the dynamic realm of CNC machining operations.

In an effort to control or minimize the impacts of both known and unknown risk factors which could interfere with the productivity and profitability of $\mathrm{CNC}$ operations by interrupting the manufacturing process, it is crucial to develop tools to predict the effects of key risk factors beforehand in order to avoid, eliminate, or minimized the consequences of such risks. The weaknesses inherent to this research are the limitations of a simulation model in comparison to actual operations. The volume of possible variations has been limited to this research case.

While the possibilities, probabilities and the impacts of risks factors may continually change over time, varying risks will have a much more profound effect on $\mathrm{CNC}$ operations if identified too late. This will, in turn make it difficult to manage the consequences of risk, may cause more damage and be much more expensive.

\section{Conclusion}

This article presents the research done on investigating risks associated with providing a quantitative method of service quality on CNC Machine Tools risk factors. The results of this research have created a method of quantifying risks associated with $\mathrm{CNC}$ machine service. Additionally, a generic novel tool has been created which is utilized for evaluating and assessing these risks. This tool provides the means of creating a quantitative measure of risk assessment to better identify and prioritize risks which enables the organization to better manage $\mathrm{CNC}$ machine down time. It is of great importance to modern organizations that use sophisticated highly engineered systems i.e., CNC Machining Centers to manage risks resulting in production interruptions. The results of this risk management process improve productivity and reduces $\mathrm{CNC}$ down time, and therefore increases output and product yields. This process also considerably improves the overall performance of the organization. CNC machine maintenance and downtime are major causes of poor quality, inefficient performance, and low productivity throughout high-tech operations. While the focus of this article is specifically on CNC machining these same methods and techniques can most certainly be applied and utilized within all highly engineered systems. A substantial number of operational interruptions are due to machine down time as a result of either scheduled or unscheduled maintenance. There is currently no systematic means of CNC service data transmission and collection as well as a lack of quantitative service quality data systems capable of delivering this information. While numerous manufacturing management systems throughout production operations have been implemented over time, none of these systems address the risks associated with poor service quality and process interruption or the minimization of operational downtime. This constitutes a management approach unrecognized in the past and will usher in a fourth generation of in the world of maintenance.

Consequently, the intention of this research is to prepare CNC service operations in recognizing risk factors as a foundation of the risk assessment \& management process and perform the analysis in the initial stages of the Risk Management process to identify and quantify risk priorities.

\section{Acknowledgements}

I would like to acknowledge all the assistance received from Paul Ranky, Professor, New Jersey Institute of Technology' Mechanical and Industrial Engineering 
department who provided insight, vision and expertise assisting with this research that greatly improved the manuscript. I also thank David Ferguson Distinguished Professor, Stony Brook University's College of Engineering and Applied Sciences for his advice and support with this research.

\section{References}

[1] Abdel-Basset, M., Mohamed, R., (2020), A novel plithogenic TOPSIS- CRITIC model for sustainable supply chain, risk management, Journal of Cleaner Production 247, 119586.

[2] Ahmed, Hani, (2017), Impact Assessment of Construction Supply Chain Risk Changes on Project Time and Cost, University of Waterloo, Civil Engineering.

[3] Aqlan, F., \& Lam, S. S. (2016). Supply chain optimization under risk and uncertainty: A case study for high-end server manufacturing. Computers \& Industrial Engineering, 93, 78-87.

[4] Baryannis, G., Validi, S., Dani, S., and Antoniou, G., (2019), Supply chain risk management and artificial intelligence: state of the art and future research directions, International Journal Of Production Research 2019 Vol. 57, No. 7, 2179-2202.

[5] Beamon Benita M. (1998) Supply chain design and analysis: models and methods. International Journal of Production Economics 1998; 55 (3): 281-94.

[6] Behzadi, Golnar, (2018). Agribusiness supply chain risk management: a review of quantitative decision models. Omega 79, 21e42. et al. 2018).

[7] Cedillo-Camposa, M. G., González-Ramírez, R. MejíaArguetac, C., González-Feliu, J., (2020), Data-driven decision making in supply chains, Computers \& Industrial Engineering, Volume 139, January 2020, 106022.

[8] Dutta, I., Dutta, S., \& Raahemi, B. (2017). Detecting financial restatements using data mining techniques. Expert Systems With Applications, 90, 374-393.

[9] Epstein, L. G. (2004). A definition of uncertainty aversion. In Uncertainty in Economic Theory (pp. 187-224). Routledge.

[10] Enyoghasi, C., Brown, A., Aydin, R., Badurdeen, F., (2019), Quantitative risk modelling for evaluating sustainable product design, International Journal of Sustainable Manufacturing.

[11] Enyoghasia, C., Badurdeena, F., (2020), Risk-informed simulation for sustainable product design decision making, 17th Global Conference on Sustainable Manufacturing, Procedia Manufacturing 43 (2020) 399-406.

[12] Franca, R. B., Jones, E. C., Richards, C. N., \& Carlson, J. P. (2010). Multi-objective stochastic supply chain modeling to evaluate tradeoffs between profit and quality. International Journal of Production Economics, 127 (2), 292-299.

[13] Gaudenzi, B. \& Borghesi, A. (2006). Managing risks in the supply chain using the AHP method. The International Journal of Logistics Management, 17 (1), 114-136. 31.

[14] Geng, R., Bose, I., \& Chen, X. (2015). Prediction of financial distress: An empirical study of listed Chinese companies using data mining. European Journal of Operational Research, 241 (1), 236-247.
[15] Green, W. 2018. "Five Lessons from the KFC Chicken Crisis." CIPS Supply Management. https://www.cips.org/supplymanagement/analysis/2018/februa ry/five-lessons-from-the-kfc-chicken-crisis/.

[16] Heckmann, I., Comes, T., \& Nickel, S. (2015). A critical review on supply chain risk - Definition, measure and modelling. Omega, 52, 119-132.

[17] Ho, W., Zheng, T., Yildiz, H., \& Talluri, S. (2015). Supply chain risk management: A literature review. International Journal of Production Research, 53 (16), 5031-5069.

[18] Karaa M. E., Firata, S. U. O., Ghadgeb, A., (2020), A data mining-based framework for supply chain risk management, Computers \& Industrial Engineering 139 (2020) 105570.

[19] Kaura, H., Singh, S. B., Garza-Reyesc, J., Mishrad, N. (2020), Sustainable stochastic production and procurement problem for resilient supply chain, Computers \& Industrial Engineering, Volume 139, January 2020, 105560.

[20] Khan, O., and B. Burnes. 2007. "Risk and Supply Chain Management: Creating a Research Agenda." The International Journal of Logistics Management 18 (2): 197-216.

[21] Merve Er Karaa, Seniye Ümit Oktay Firata, Abhijeet Ghadgeb, (2020). A data mining-based framework for supply chain risk management Computers \& Industrial Engineering 139,105570 .

[22] Nagurney, A., Masoumi, A. H., \& Yu, M. (2012). Supply chain network operations management of a blood banking system with cost and risk minimization. Computational Management Science, 9 (2), 205-231.

[23] Snyder, L. V., Z. Atan, P. Peng, Y. Rong, A. J. Schmitt, and B. Sinsoysal. 2016. "OR/MS Models for Supply Chain Disruptions: A Review." IIE Transactions (Institute of Industrial Engineers) 48 (2): 89-109.

[24] Svensson, G. (2002). A conceptual framework of vulnerability in firms inbound and outbound logistics flows. International Journal of Physical Distribution \& Logistics Management, 32 (2), 110-134.

[25] Saaty, T. L. (1990). How to make a decision: the analytic hierarchy process. European journal of operational research, 48 (1), 9-26.

[26] Tobback E., Bellotti, T., Moeyersoms, J., Stankova, M., \& Martens, D. (2017). Bankruptcy prediction for SMEs using relational data. Decision Support Systems, 102, 69-81.

[27] Vilko, J., P. Ritala, and J. Edelmann. 2014. "On Uncertainty in Supply Chain Risk Management. "International Journal of Logistics Management 25 (1): 3-19.

[28] Vivas, R. C., Sant'Anna, A., Oliveira, K., Freires, F., (2020), Integrated method combining analytical and mathematical models for the evaluation and optimization of sustainable supply chains: A Brazilian case study, Computers \& Industrial Engineering Volume 139, January 2020, 105670.

[29] Zhang, Q., Shah, N., Wassick, J., Helling, R., \& van Egerschot, P. (2014). Sustainable supply chain optimisation: An industrial case study. Computers \& Industrial Engineering, 74 , $68-83$. http://www.sciencedirect.com/science/article/pii/S0360835214 001478 . 